\title{
PRIMARY EWING SARCOMA OF THE SINONASAL TRACT- A CASE REPORT
}

\author{
Nanditha Sheshanna1, Varaprasad B. Muniraju², Jyothi A. Raj33, Jyothi Prakash C4, Prashanth Veerabhadraiah ${ }^{5}$
}

1 Postgraduate Student, Department of Pathology, Raja Rajeswari Medical College, Bengaluru. 2Postgraduate Student, Department of Pathology, Raja Rajeswari Medical College, Bengaluru.

3 Professor, Department of Pathology, Raja Rajeswari Medical College, Bengaluru.

4 Professor, Department of Pathology, Raja Rajeswari Medical College, Bengaluru.

${ }^{5}$ Assistant Professor, Department of Otorhinolaryngology, Raja Rajeswari Medical College, Bengaluru.

HOW TO CITE THIS ARTICLE: Sheshanna N, Muniraju VB, Raj JA, et al. Primary Ewing sarcoma of the sinonasal tract-a case report. J. Evolution Med. Dent. Sci. 2018;7(06):809-812, DOI: 10.14260/jemds/2018/184

\section{PRESENTATION OF CASE}

A 21-year-old male presented with swelling in right medial canthus for 5 months. The swelling was soft to firm, nontender and immobile, measuring $2.5 \mathrm{cms} \times 1.5 \mathrm{cms}$.

On local examination surface was nodular on medial part without redness, scar or puncta. The nodule was seen extending superiorly up to the level of medial end of the right eyebrow, inferiorly to $5 \mathrm{~mm}$ below the level of right lower eyelid, medially close to the midpoint of the nasion and laterally upto the medial canthus of right eye till the right eyebrow [Figure 1]. With a preliminary diagnosis of dacryocystocele, further investigations were ordered.

Ewing sarcoma is a small round cell malignant neoplasm, considered to be of neuroectodermal derivation. It is relatively uncommon accounting for $6 \%-8 \%$ of primary malignant bone tumours and the second most common sarcoma in bone and soft tissue in children. ${ }^{1}$ James Ewing reported a round cell neoplasm in the radius of a 14-year-old girl in 1921. Angervall and Enzinger in 1975 described the first Ewing's Sarcomas arising in soft tissue (extraskeletal ES). Seemayer et al and Askin et al described malignant small cell tumours arising in the soft tissues having histologic features similar to peripheral neuroectodermal tumours (PNET), but with unique clinico-pathologic profile. With the advent of immunohistochemical, cytogenetic and molecular genetic techniques, these tumours are universally regarded to represent ends of a morphologic spectrum known as ES/PNET family of tumours. ${ }^{2}$

Ewing Sarcoma (ES) has both skeletal and extraskeletal forms. ${ }^{3}$ The extraskeletal form usually occurs in the soft tissues of lower extremities, paravertebral tissues, chest wall and retroperitoneum. ${ }^{4}$ Primary Ewing Sarcoma of the head and neck region is extremely rare and accounts for only 4\%$9 \%$ of all Ewing Sarcomas with a slight male preponderance.2,5 Extraskeletal Ewing Sarcoma in the sinonasal tract is so rare that only a few case reports have been published in the world literature. ${ }^{5}$

'Financial or Other Competing Interest': None.

Submission 18-12-2017, Peer Review 19-01-2018,

Acceptance 27-01-2018, Published 05-02-2018.

Corresponding Author:

Dr. Jyothi A. Raj,

Professor,

Department of Pathology,

Raja Rajeswari Medical College,

Bengaluru-560074.

E-mail: jyoki255@yahoo.co.in

DOI: $10.14260 /$ jemds $/ 2018 / 184$

\section{(c) $(1)$}

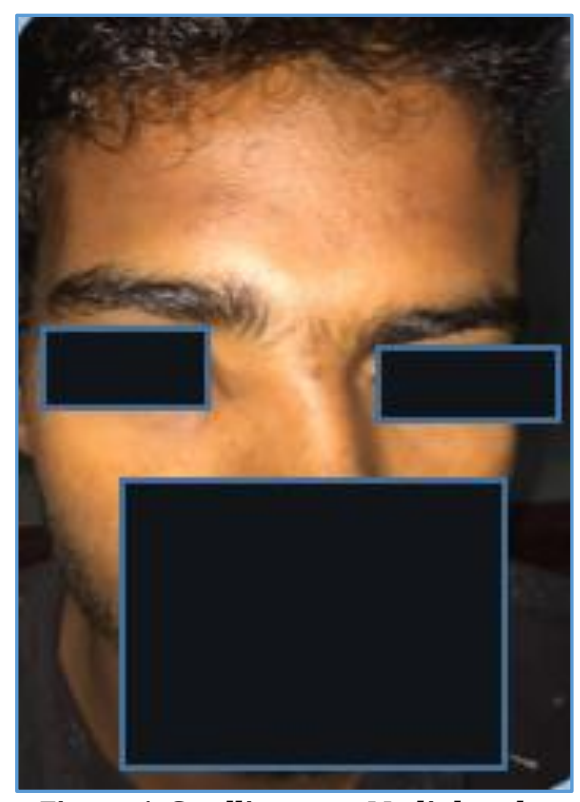

Figure 1. Swelling seen Medial to the Medial Canthus of the Right Eye

Diagnostic nasal endoscopy revealed a boggy swelling near the anterior attachment of middle turbinate, extending over the inferior turbinate. No significant posterior extension was seen. Middle meatus was not accessible. A CT scan revealed the mass close to the medial canthus of the right eye, extending upto the superior meatus, medial wall of orbit and anterior skull base [Figure 2].

\section{CLINICAL DIAGNOSIS}

With the above radiologic findings, malignancy of right lacrimal sac was suspected and FNAC advised.

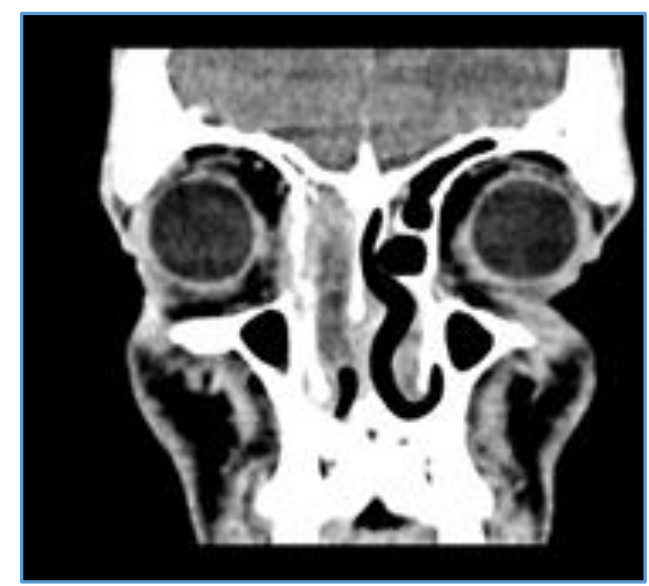

Figure 2. CT PNS shows a Soft Tissue Mass Close to the Medial Canthus of the Right Eye 


\section{Cytology}

Blood mixed material was aspirated. Smears were cellular, showed small blue round cells, in clusters and dispersed singly. Occasional binucleated cells were noted, which prompted a cytology diagnosis of lymphoproliferative disorder [Figure 3]. Biopsy was advised for histopathologic examination.

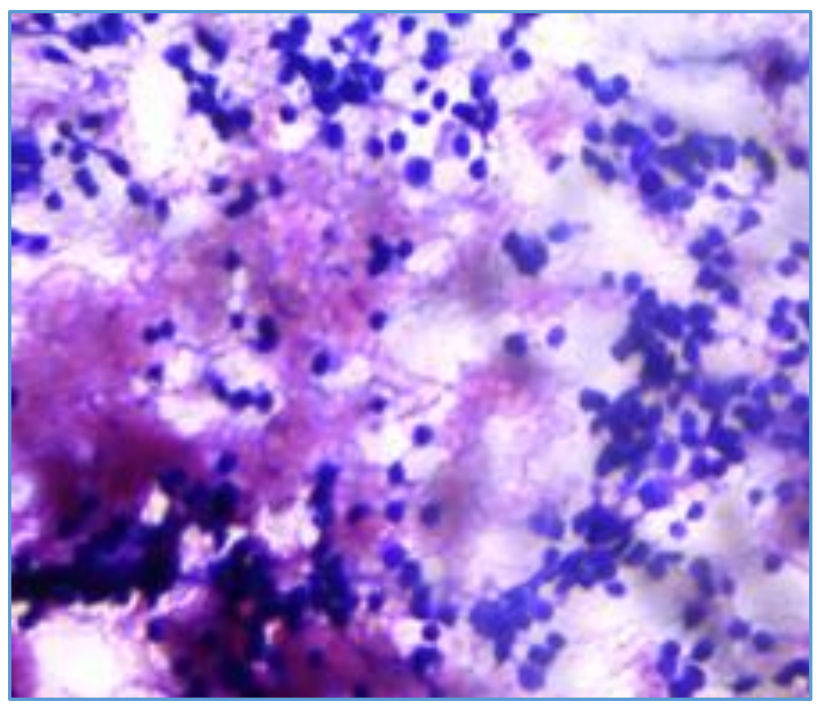

Figure 3. Smears show Small Blue Round Cells in Loose Clusters [MGG, 10x]

\section{Histopathology}

Gross: Container 1 labelled tissue from orbital part of the tumour: A single grey white soft tissue bit measuring $1 \times 0.5 \times 0.5 \mathrm{~cm}$. Container 2 labelled tissue from nasal part of tumour: 3 grey white soft tissue bits, together measuring $1 \times 0.5 \times 0.5 \mathrm{~cm}$, cut surface grey white with mucoid areas.

Microscopy: Sections showed a diffusely infiltrating neoplasm forming cords and clusters with mild crush artefact. The neoplastic cells were small and round with scant cytoplasm, high N: C ratio and hyperchromatic nuclei. The cells lacked pleomorphism. Structures resembling rosettes were noted. The tumour was seen infiltrating skeletal muscle and adipose tissue [Figures 4 to 6]. A morphologic diagnosis of small round cell tumour, possibly olfactory neuroblastoma in the right nasal cavity was made, bearing in mind the age of the patient, location of the tumour and the presence of rosettelike structures on microscopy. Immunohistochemistry was advised for confirmation.

\section{Immunohistochemistry}

A panel of immunohistochemical markers were applied considering the differential diagnoses of small round cell tumours likely to arise in this location. The tumour cells in the present case were MIC2 positive and LCA, CK, desmin and synaptophysin negative [Figure 7]. Ki-67 index was 25\%.

\section{FINAL DIAGNOSIS}

Correlating IHC with morphology, a diagnosis suggestive of Ewing Sarcoma in the right paranasal sinus was given and FISH was advised for EWSR1 rearrangement.

The patient could not afford FISH and preferred to go ahead with the treatment available.

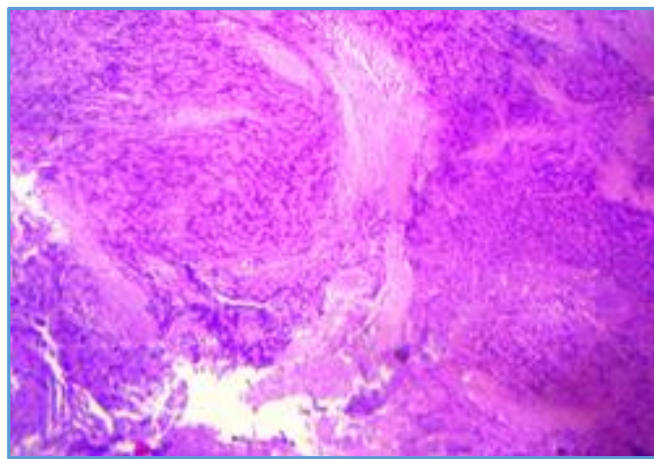

Figure 4. Shows a Diffuse Neoplasm Infiltrating in the Form of Cords and Clusters showing Mild Crush Artefact [4X, Hand E]

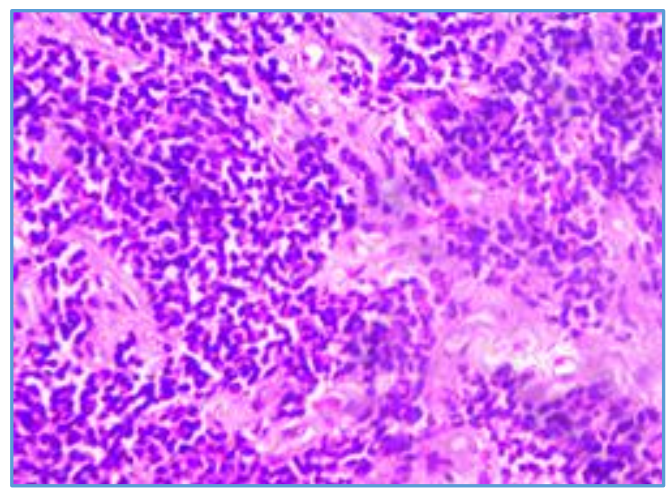

Figure 5. Small and Round Neoplastic Cells with High N:C Ratio, Hyperchromatic Nuclei and Scant Cytoplasm [40x, $H$ and $E$ ]

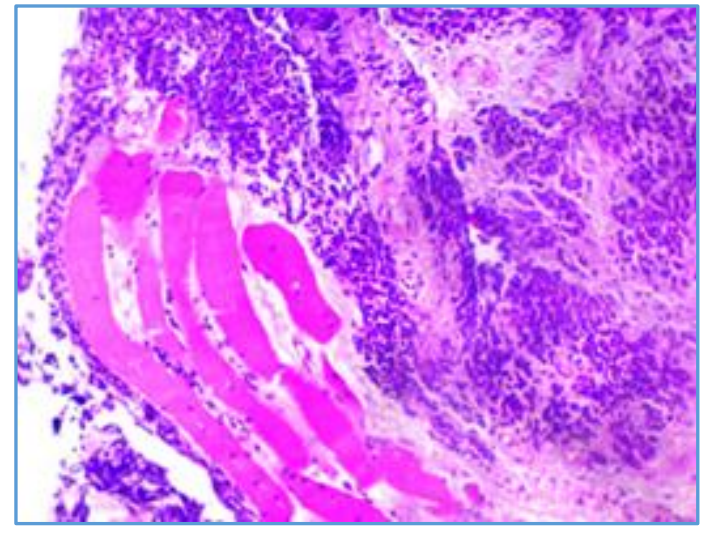

Figure 6. Tumour seen Infiltrating Skeletal Muscle [10x, $\mathrm{H}$ and $\mathrm{E}]$

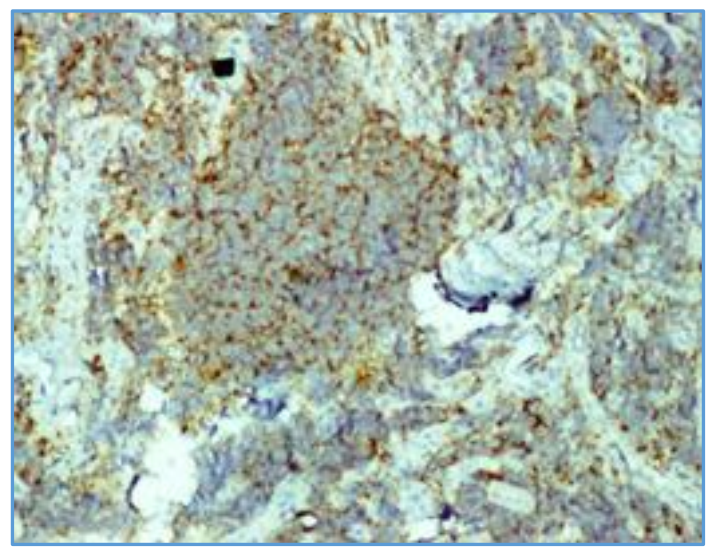

Figure 7. CD99 Positivity in Tumour Cells [20x] 


\section{DISCUSSION}

Ewing's family of tumours (EFT) are malignant neoplasms affecting children and young adults, mostly arising in the long bones or pelvis. Primary EFT of head and neck is uncommon and primary sinonasal EFT is even rarer. 6

EFT is currently listed in the WHO blue book for head and neck tumours as a sinonasal neuroectodermal tumour with a presumed neuroectodermal cell origin. It is listed separately from sinonasal bone tumours, implying a mucosal or extraosseous origin. Previous cases in the literature have also been specifically designated as "extraskeletal" Ewing sarcoma of the nose, nasal fossa or sinus.6,7 These tumours are rarely seen associated with other mucosal sites such as lower respiratory, urologic, gynaecologic and gastrointestinal tracts.

A range of poorly-differentiated small blue round cell neoplasms arise from the sinonasal tract including poorlydifferentiated carcinomas, lymphoma, rhabdomyosarcoma, melanoma, olfactory neuroblastoma and EFT. EFTs are the rarer ones among these tumours in this location, and have not been extensively reported in literature. ${ }^{6}$ Differential diagnoses of these tumours based on morphology alone is complicated by the fact that many of them show similar histologic features and immunophenotypic overlap. ${ }^{8}$

Epithelial small round blue cell tumour in this location is usually the poorly differentiated non-keratinising squamous cell carcinoma involving the maxillary sinus followed by nasal cavity. In its poorly differentiated form, it is most difficult to distinguish from other undifferentiated small round cell tumours. Carcinomas and olfactory neuroblastoma are particularly difficult to differentiate from EFTs also, because of the presence of nesting pattern of cells in many of these cases. Carcinomas are generally diffusely positive for cytokeratins and generally not seen in young patients unlike EFTs.

Olfactory neuroblastoma is a neuroectodermal tumour thought to arise from neuronal precursors, usually involving the superior nasal cavity/ cribriform plate. Presence of fibrillary cell process is a typical feature in them. Nesting, lack of keratin positivity and occurrence in young patients are features common to both olfactory neuroblastoma and EFT. They are different from EFT by being positive for S100 and neuroendocrine markers [synaptophysin, NSE] and negative for CD-99/ MIC2.

Sinonasal melanoma is characterised by the presence of melanin pigmentation and prominent eosinophilic nucleoli. It is positive for S100, HMB-45 and Melan-A on IHC. Embryonal rhabdomyosarcoma is common in children, has small cells distributed in cambium layer and rhabdomyoblasts may be seen. It is ruled out with IHC for Desmin, myogenin and Myo D1. Occasional cases of EFT are focally positive for S100 or Desmin, never for the other markers. ${ }^{6}$ Lymphomas are CD-45 and LCA positive on IHC.

EFTs are common in younger patients, can occur anywhere in the sinonasal tract, most often involving at least one sinus and almost $100 \%$ of cases are positive for MIC2/ CD-99. The case discussed here was MIC2 positive and negative for CK, LCA, desmin and synaptophysin, suggestive of Ewing Sarcoma.

\section{Cytogenetics}

Cytogenetics and molecular techniques such as FISH and RTPCR, are useful tools in the diagnosis of difficult cases among this group of neoplasms. However, they are not prerequisites for diagnosis. ${ }^{6}$ Majority of patients have EWS- FLI1 fusion transcript from translocation between 3 ` end of FLI1 gene on 11q24 and 5 end EWS gene on 22q12. 10\% - 15\% of cases have t (21:22) (q22: q12).

Hafezi $S$ et al 6 have presented the largest series of sinonasal EFT to date, most of which have molecular confirmation. EFT appears to be less aggressive in this location than the more typical extremity bone and soft tissue tumours; however, the limited follow-up in the literature precludes a definitive statement on behaviour of EFT in this site. Hafezi $\mathrm{S}$ et al express surprise at a less aggressive clinical course, given the complex anatomy of the primary site, difficulty in achieving a negative-margin resection, late presentation and early access by the tumour to vital structures.

Multimodality therapy (multidrug chemotherapy, RT, surgical resection) is indicated for EFTs. They have a propensity for haematogenous and lymphatic spread. ${ }^{9}$ Advances in treatment have improved survival rates. Those with metastasis at presentation have poorer outcomes. Inability to achieve complete resections and negative margins in tumours of the sinonasal tract confers a worse prognosis for these tumours. ${ }^{6}$

\section{CONCLUSION}

Malignancy arising in the nasal cavity and paranasal sinus are heterogeneous. EFTs in this region show great diversity, similar to these tumours at other sites. This location is particularly problematic owing to the large number of potential mimics including epithelial, mesenchymal and neural tumours, most of which are more common than EFT. Accurate diagnosis is challenging due to overlapping clinical, radiographic and histopathologic features. Advances in molecular genetics and appropriate use of IHC greatly assist in the differential diagnosis, in combination with radiographic and clinical correlation.

\section{REFERENCES}

[1] Ushigome S, Machinami R, Sorensen PH. Ewing sarcoma/Primitive Neuro Ectodermal Tumour (PNET). Chapter 14. In: Fletcher CDM, Unni KK, Mertens F. eds. World Health Organization Classification of tumours. Pathology and genetics of tumours of soft tissue and bone. Lyon, France: IARC Press 2002:298-300.

[2] Weiss SW, Goldblum JR. Ewing's sarcoma/ PNET tumor family and related lesions. Chapter 31. In: Enzinger \& Weiss's soft tissue tumors. $5^{\text {th }}$ edn. China: Mosby Elsevier 2008:945-87.

[3] Iezzoni JC, Mills SE. Undifferentiated small round cell tumors of the sinonasal tract: differential diagnosis update. Am J Clin Pathol 2005;(Suppl 124):S110-21.

[4] Gupta S, Gupta OP, Mehrotra S, et al. Ewing sarcoma of the maxilla: a rare presentation. Quintessence Int 2009;40(2):135-40.

[5] Yeshvanth SK, Ninan K, Bhandary SK, et al. Rare case of extraskeletal Ewings sarcoma of the sinonasal tract. J Can Res Ther 2012;8(1):142-4.

[6] Hafezi S, Seethala RR, Stelow EB, et al. Ewing's family of tumors of the sinonasal tract and maxillary bone. Head and Neck Pathol 2011;5(1):8-16. 
[7] Wenig BM, Dulguerov P, Kapadia SP, et al. Neuroectodermal tumors. In: Barnes EL, Eveson JW, Reichart P, et al. eds. World Health Organization classification of tumours. Pathology and genetics of head and neck tumours. Lyon, France: IARC Press 2005:65-70.

[8] Wenig BM. Undifferentiated malignant neoplasms of the sinonasal tract. Arch Pathol Lab Med 2009;133(5):699-712.
[9] Brandwein-Gensler M. Sinonasal and nasopharyngeal surgical pathology. Section 6. Chapter 15. Upper respiratory system. In: Silverberg SG. edr in chief. Silverberg's Principles and practice of surgical pathology and cytopathology. Vol 1. 4th edn. China: Churchill Livingstone Elsevier 2006:806-7. 